\title{
Intracranial Aneurysm Rupture Following Intravenous Thrombolysis for Stroke
}

\author{
Faizal Haji, Brian van Adel, Michael Avery, Joseph Megyesi, G. Bryan Young
}

Can J Neurol Sci. 2014; 41: 95-98

Treatment of acute ischemic stroke with intravenous tissue plasminogen activator (IV-tPA) has proven efficacy and safety up to 4.5 hours from clinical onset. ${ }^{1}$ Treatment with IV-tPA may lead to potentially serious complications, the most devastating being intracranial hemorrhage (ICH). In this setting, ICH risk may be increased by advanced age, hypertension, large infarction volume, anticoagulant use, previous stroke, and cerebral vascular lesions. Current guidelines cite many of these factors, including the presence of unruptured intracranial aneurysms (UIAs), as exclusion criteria for thrombolytic therapy for acute ischemic stroke. ${ }^{2}$

The population affected by these guidelines is not trivial: among patients with ischemic stroke, the prevalence of UIAs is between 6.6-9.3\%, noticeably higher than the general population. ${ }^{2}$ Thus, up to one in every ten patients receiving thrombolysis for acute stroke may harbor an UIA; a number that is likely to grow with advances in stroke imaging, such as computed tomogram (CT) and magnetic resonance (MR) angiography (CTA/MRA). This compounds the therapeutic dilemma created by the paucity of evidence (and subsequent controversy) regarding whether IV thrombolysis truly increases the likelihood of aneurysm rupture. Thus, "is thrombolysis in the presence of an unruptured intracranial aneurysm safe?" remains an important unanswered question, with attendant, potentially life-threatening risk, for a significant proportion of ischemic stroke patients.

To date, two cases of intracranial aneurysm rupture following intravenous thrombolytic therapy have been reported: one in the setting of acute myocardial infarction, the other in a patient with symptoms of sentinel hemorrhage who received IV-tPA for ischemic stroke. ${ }^{3,4}$ We report the case of a previously asymptomatic patient with multiple UIAs who suffered massive (and ultimately fatal) subarachnoid hemorrhage (SAH) following IV-tPA for acute ischemic stroke. To our knowledge, this is the first reported case where a previously asymptomatic intracranial aneurysm was the likely cause of a massive subarachnoid hemorrhage following intravenous thrombolysis for acute ischemic stroke.

\section{Case Report}

\section{History}

A 71-year-old right-handed-male presented to hospital within thirty minutes of a witnessed sudden onset right face and arm hemiparesis, right-sided neglect and global aphasia. His medical history included pneumonectomy for lung cancer, peripheral arterial disease with bilateral aortofemoral bypass, hypertension, hyperlipidemia, and 30-pack-years of smoking. The patient was not receiving antiplatelet therapy. Examination revealed blood pressure of $150 / 80 \mathrm{mmHg}$, heart rate of 70 and an initial National Institutes of Health Stroke Scale (NIHSS) score of 15. Laboratory investigations were within normal limits. The admission non-contrast cranial CT scan showed a hyperdense MCA, an Alberta Stroke Program Early CT Score (ASPECTS) of 8 and no hemorrhage or mass lesion (Figure 1).

\section{Management}

The patient was assessed by the Ontario Telemedicine Network Telestroke protocol. As no contraindications for thrombolysis were identified, the patient received $90 \mathrm{mg}$ of IVtPA in standard fashion within three hours of symptom onset. He remained hemodynamically and clinically stable during the infusion of IV-tPA. The patient recovered some neurological function over the next few hours, with mild improvement of his aphasia (vocalizing single words). He was admitted to intensive care for monitoring.

\section{Clinical Course}

Approximately five hours post-thrombolysis, the patient's level of consciousness rapidly deteriorated. His blood pressure increased to 200/90 $\mathrm{mmHg}$ and his Glasgow Coma Scale fell from 14 to 10 (E3V1M6), with development of right-sided hemiplegia. An emergent CT scan demonstrated a diffuse Fischer III SAH and early signs of hydrocephalus (Figure 2). Despite aggressive medical management, he progressed to decorticate coma. The patient was intubated and transferred emergently to a tertiary care facility for neurosurgical assessment, where an external ventricular drain was placed. Computed tomogram angiography (CTA) demonstrated severe atherosclerotic stenosis of the proximal left internal carotid artery (ICA) and a dissection of the left extracranial ICA, just distal to the segment of stenosis (Figure 3). Based on the imaging findings, we suspect the acute dissection of the left ICA precipitated a large artery-to-artery thromboembolism, resulting in the patient's initial presentation of proximal left MCA

\footnotetext{
From the Department of Clinical Neurological Sciences (FH, JM, GBY), University of Western Ontario, London; Division of Neurology (BvA), Neurosurgery and Diagnostic Imaging, McMaster University, Hamilton, Ontario; Division of Neurosurgery (MA), University of Calgary, Calgary, Alberta, Canada.

Received June 3, 2013. Final Revisions Submitted August 2, 2013.

Correspondence to: Faizal Haji, University Hospital, London Health Sciences Centre, Room B7-005, 339 Windermere Road, London, Ontario, N6A 5A5, Canada. Email: fhaji@uwo.ca.
} 

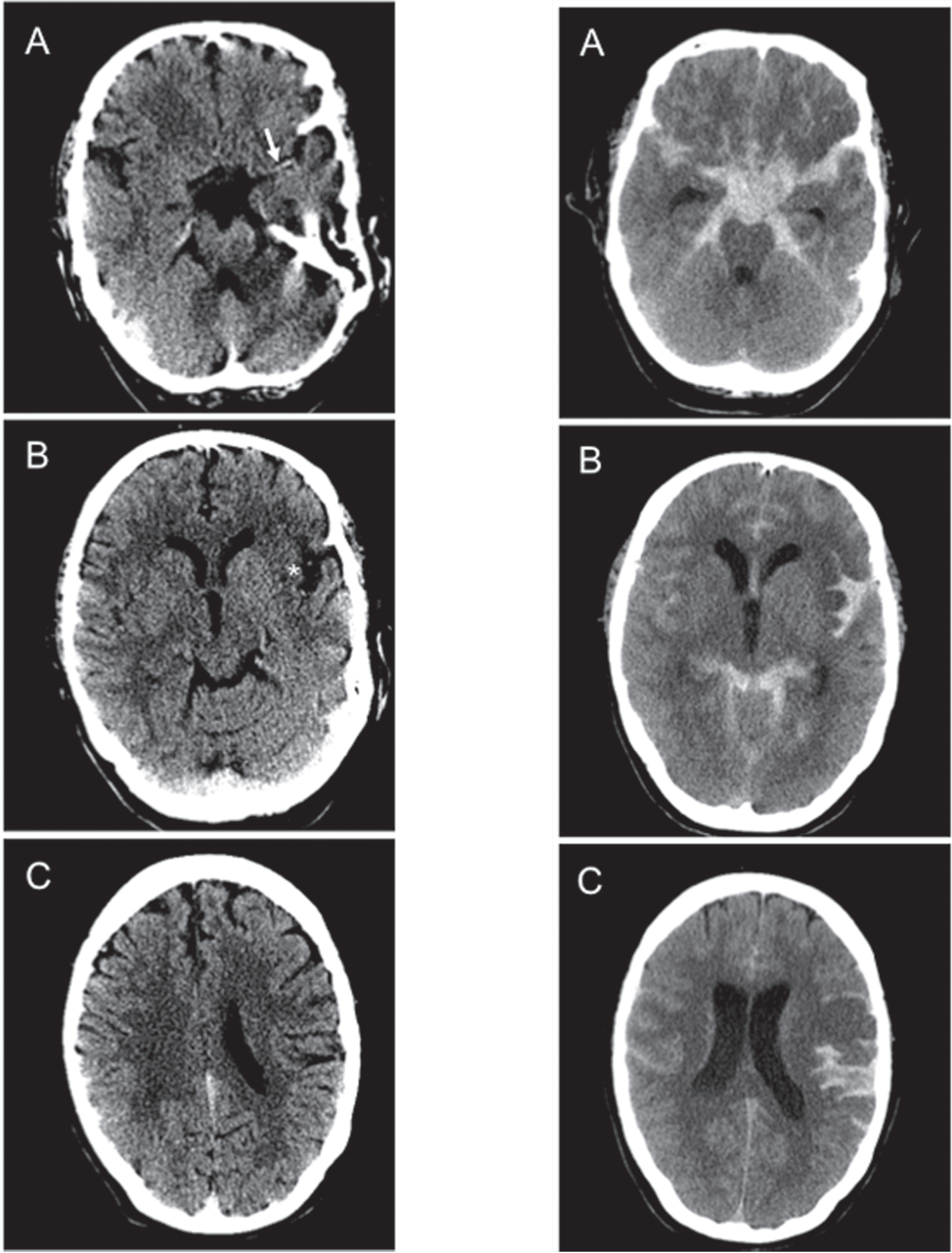

Figure 1: A-C: Admission cranial non-contrast computer tomography (NCCT) scan performed as part of an acute stroke protocol. A: Axial CT image demonstrates a left hyperdense MCA sign (arrow). $B$ and $C$ : ischemic changes in the left insular cortex and left frontal lobe. $A S P E C T S=8$. No evidence of mass lesion, or hemorrhage.
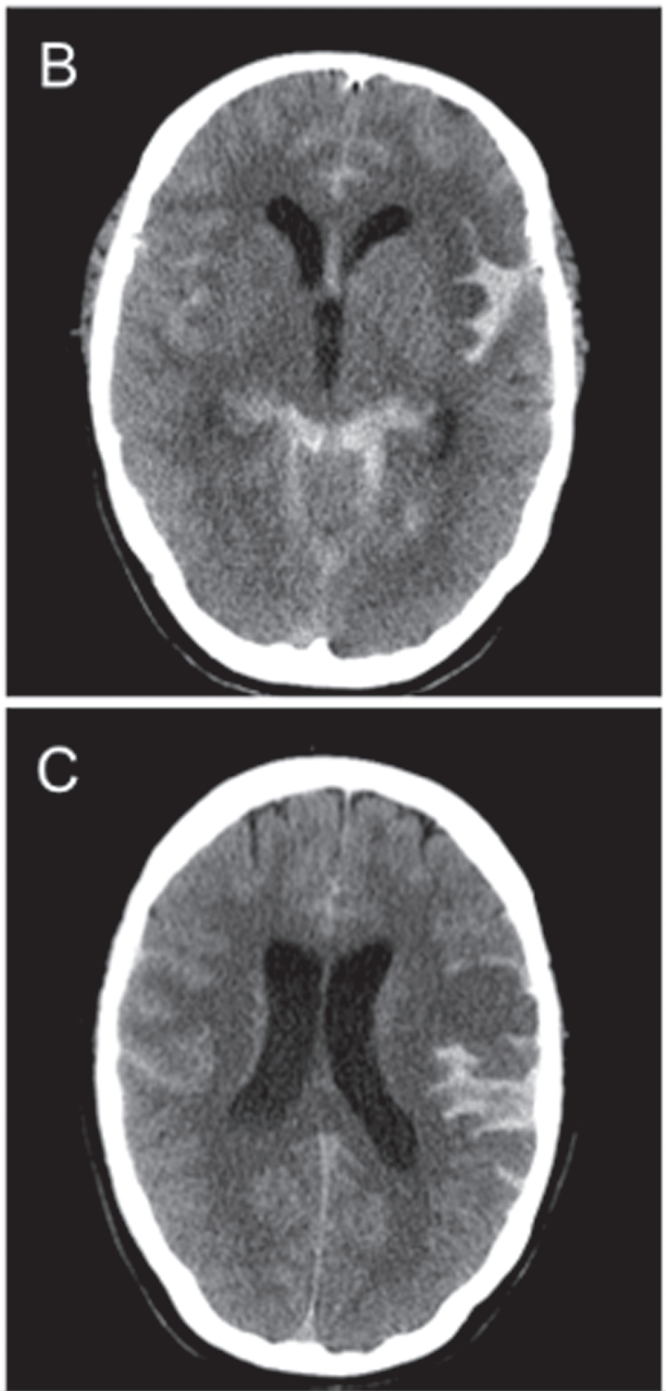

Figure 2: A repeat cranial non-contrast computer tomography (NCCT) approximately 5 hours after treatment with IV-tPA for the patient's acute left MCA ischemic stroke. A-C: Axial CT scan demonstrates a diffuse, Fisher grade III, subarachnoid hemorrhage (SAH) and further evolution of the patient's left MCA infarct. 

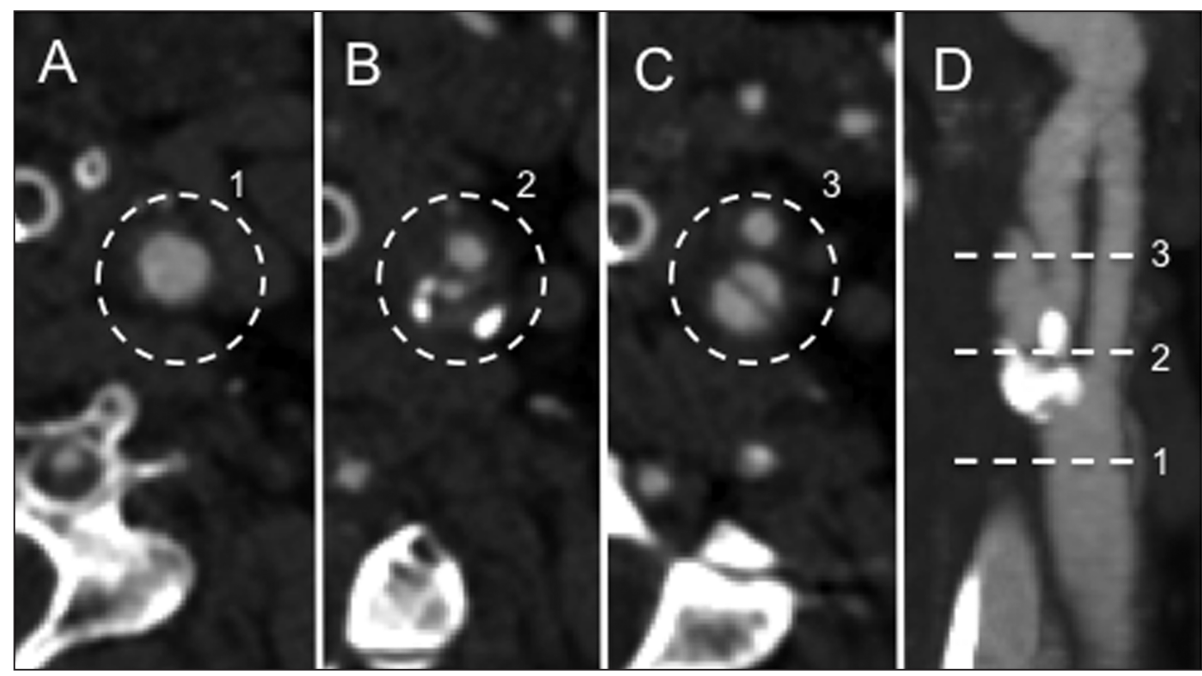

Figure 3: Computed tomogram angiogram 12 hours from onset of the acute ischemic stroke presentation. A-D: Left carotid artery shows normal caliber of the distal common carotid artery (A), and severe stenosis of the proximal internal carotid artery (ICA) just beyond the carotid bifurcation $(B)$, and a dissection of the more distal extracranial ICA (C). D: Corresponding maximum intensity projection (MIP) imaging of the left carotid artery demonstrating a dissection flap of the left ICA. 1-3 represents the anatomical level of the left carotid artery shown in the axial source images from $A-C$.

occlusion. Computed tomography angiography also revealed saccular aneurysms of the left posterior communicating (Pcomm) artery and right posterior inferior cerebellar artery (PICA), measuring $4 \mathrm{~mm}$ × $6 \mathrm{~mm}$ and $6 \mathrm{~mm} \times 8.5 \mathrm{~mm}$ respectively (Figure 4). Despite ongoing CSF diversion, the patient's neurological status did not improve. After discussion with the family, supportive measures were withdrawn and the patient died.

\section{Discussion}

Despite the extended time window for intravenous thrombolysis, ${ }^{1}$ the majority of acute stroke patients do not receive IV-tPA either due to delayed presentation or failure to meet inclusion/exclusion criteria. ${ }^{2,5}$ However, as our experience grows, the results from inadvertent or deliberate off-label use of thrombolysis has called some of these criteria into question. For instance, among published reports of 34 patients with asymptomatic intracranial aneurysms who received IV thrombolysis for acute ischemic stroke, ${ }^{2,5,6}$ none suffered SAH attributable to aneurysm rupture following administration of IVtPA. Collectively, this literature supports the claim that, among patients with small $(<10 \mathrm{~mm})$ asymptomatic aneurysms, IV-tPA for acute ischemic stroke may be administered without additional adverse events. ${ }^{2,6}$

To our knowledge, only two cases of aneurysm rupture following intravenous thrombolysis have been reported. In the first, ${ }^{3}$ the combination of high-dose IV-tPA $(100 \mathrm{mg})$ and the use of a heparin infusion for treatment of acute myocardial infarction may have increased the likelihood of aneurysm rupture. ${ }^{6}$ Thus, some have argued that there is no scientific basis for the current exclusion of patients with asymptomatic aneurysms in the setting of acute ischemic stroke, ${ }^{5}$ where the dose of IV-tPA is lower

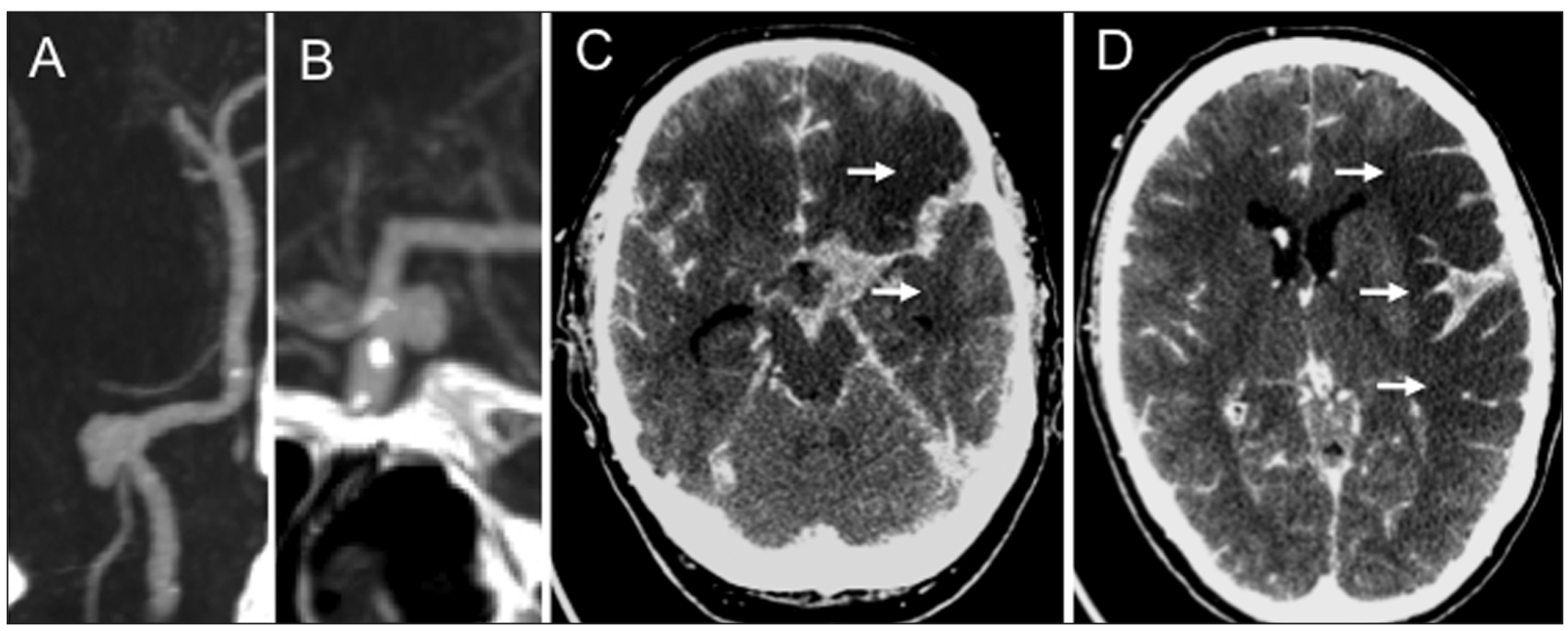

Figure 4: Repeat $C T$ head and CT angiogram 12 hours from onset of the acute ischemic stroke presentation A-B: CT angiography identified two intracranial aneurysms involving the right PICA (A) and left PComm (B). C-D: Further evolution of the large left MCA territory cerebral infarction as noted by marked hypodensity (arrows). 
$(0.9 \mathrm{mg} / \mathrm{Kg}$ vs. $1.1 \mathrm{mg} / \mathrm{Kg})$. The second reported case involved a patient whose history suggested a sentinel hemorrhage three days prior to presentation with ischemic stroke, which was not ascertained prior to administration of IV-tPA. ${ }^{4}$ The subsequent SAH was likely the result of re-bleeding precipitated by tPA dissolution of the thrombus within the dome of a previously ruptured aneurysm. ${ }^{2}$ As the authors note, ${ }^{4}$ had this history been available at the time of thrombolysis the treatment would have been withheld.

The current report, however, gives reason for pause. In our case, a standard dose of IV-tPA was administered in the setting of small $(<10 \mathrm{~mm})$ asymptomatic aneurysms, with subsequent massive subarachnoid hemorrhage. Although both cerebral infarction and SAH may be seen in the setting of acute intracranial arterial dissection, ${ }^{7}$ in this case the patient's observed arterial stenosis and dissection were exclusively extracranial. Thus, although results of a post-mortem are not available to confirm that the SAH was caused by aneurysm rupture, given the patient's clinical course, imaging, and pattern of hemorrhage we favour this to be the most likely etiology.

A number of pathophysiological mechanisms have been documented which may explain the outcome observed in this case. Specifically, hypertension that accompanies the ischemiaassociated acute stress response, ${ }^{4}$ as well as tPA-induced hemodynamic changes, have been associated with increased turbulence within the cerebral vasculature. ${ }^{3}$ In turn, pathological studies have demonstrated that increased turbulence and shear stress inside an aneurysm sac may precipitate rupture, ${ }^{8}$ even among previously asymptomatic lesions. Furthermore, tPA is known to increase the risk of intracerebral hemorrhage by altering vascular permeability and the integrity of the vascular basal lamina at the site of vascular pathology. ${ }^{6}$ Although far from conclusive, it is conceivable that such local angioarchitectural and physiological changes induced by ischemia and subsequent tPA administration may have incited aneurysm rupture, given the temporal relationship between the ischemic event, fibronolytic therapy, and subsequent SAH in this case. However, it is also known that not all aneurysms are at equal risk of rupture; in the present case, the multiplicity, size $(>7 \mathrm{~mm})$ and location (posterior circulation) of the lesions increased the overall risk of rupture, independent from ischemic stroke or IV-tPA factors. It is difficult to determine the exact impact that these additional risk factors, as well as the patient's elevated blood pressure, had in our case.

In the two previous reports ${ }^{3,4}$ and the current case where massive SAH likely resulted from aneurysmal rupture following IV-tPA, the ensuing hemorrhage resulted in progression to death or withdrawal of care. While the presence of a significant ischemic insult, advanced patient age and other factors contributed to the patients' demise in these cases, it is clear that the SAH also played a significant role. However, it is not the intent of the authors to suggest that IV-thrombolysis should be withheld in the setting of UIAs based on current evidence; a single case report is not sufficient to make such a recommendation and would exclude a large cohort of ischemic stroke patients from receiving lifesaving treatment. Even in our case, had the history of UIA been known we would have informed the patient's family of the increased risk of hemorrhage and, given the size of the patient's infarct, a decision to proceed with thrombolysis may have been made regardless. In addition, more aggressive lowering of blood pressure below the current guidelines $(<185 / 110)$ may have been considered. Thus, in the end, therapeutic decisions will need to be made on a case-bycase basis, however these decisions can and should be evidencebased. As such, we would advocate for ongoing research to generate further safety data on the use of IV-tPA for acute ischemic stroke in patients with asymptomatic UIAs, especially with respect to risk stratification based on aneurysm size and location. To do so, we support establishing an ongoing, multicentre registry of acute ischemic stroke patients given thrombolysis who harbour UIAs ${ }^{9}$ to better define the true risk of intracranial hemorrhage in this unique cohort of patients.

\section{Conclusion}

The use of intravenous thrombolysis for acute ischemic stroke in the presence of asymptomatic UIAs remains controversial. Although an increasing number of patients have been successfully treated with IV-tPA in this setting, the results of this report suggest that caution is still warranted. Further data is needed to help guide therapeutic decisions for this small, but significant population of stroke patients.

\section{ACKNOWLEDGEMENTS}

The authors wish to acknowledge the contributions of Dr. Donald Lee's in preparing and reviewing the figures presented in this report.

\section{REFERENCES}

1. Hacke W, Kaste M, Bluhmki E, et al. Thrombolysis with alteplase 3 to 4.5 hours after acute ischemic stroke. N Engl J Med. 2008; 359(13):1317-29.

2. Edwards NJ, Kamel H, Josephson SA. The safety of intravenous thrombolysis for ischemic stroke in patients with pre-existing cerebral aneurysms: a case series and review of the literature. Stroke. 2012 Feb;43(2):412-6.

3. Lagares A, Gomez P, Lobato R, Alen J, Campollo J, Benito-Leon J. Cerebral aneurysm rupture after r-tPA thrombolysis for acute myocardial infarction. Surg Neurol. 1999;52:623-6.

4. Rammos SK, Neils DM, Fraser K, Klopfenstein JD. Anterior communicating artery aneurysm rupture after intravenous thrombolysis for acute middle cerebral artery thromboembolism: case report. Neurosurgery. 2012;70(6):1603.

5. Aleu A, Mellado P, Lichy C, Kohrmann M, Schellinger PD. Hemorrhagic complications after off-label thrombolysis for ischemic stroke. Stroke. 2007;18;38(2):417-22.

6. Kim J-T, Park M-S, Yoon W, Cho K-H. Detection and significance of incidental unruptured cerebral aneurysms in patients undergoing intravenous thrombolysis for acute ischemic stroke. J Neuroimaging. 2012;22(2):197-200.

7. Alotaibi NM, Fugate JE, Kaufmann TJ, Rabinstein AA, Wijdicks EFM, Lanzino G. Intracranial supraclinoid ICA dissection causing cerebral infarction and subsequent subarachnoid hemorrhage. Neurocrit Care. 2013;18(2):252-6.

8. Ferguson GG. Physical factors in the initiation, growth, and rupture of human intracranial saccular aneurysms. J Neurosurg. 1972;37 (6):666-77.

9. Desai JA, Jin AY, Melanson M. IV thrombolysis in stroke from a cavernous carotid aneurysm to artery embolus. Can J Neurol Sci. 2011 Mar;38(2):352-3. 